\title{
Clinical Features and Outcomes in ESBL-Producing Microorganisms in Renal Transplant Recipients with Urinary Tract Infections
}

\author{
Joya Singh ${ }^{1}$, Mikhail de Jesus ${ }^{1}$, Leigh Cooper ${ }^{1}$, Judith Pozzerle ${ }^{2}$, Suresh J. Antony ${ }^{1,{ }^{*}}$ and Bleu Knight ${ }^{1}$ \\ ${ }^{1}$ Department of Medicine, Burrell College of Osteopathic Medicine, Las Cruces, United States \\ ${ }^{2}$ Department of Transplant Services, Las Palmas Medical Center, El Paso, United States \\ "Corresponding author: Department of Medicine, Burrell College of Osteopathic Medicine, Las Cruces, United States. Email: suresh.antony@att.net
}

Received 2019 July 17; Revised 2019 September 17; Accepted 2019 September 28.

\begin{abstract}
Multidrug-resistant organisms have become an increasing challenge in the management of both solid and non-solid organ transplants. This is especially true with extended spectrum beta-lactamases (ESBL) and carbapenem-resistant Enterobacteriaceae (CRE) infections. We performed a retrospective study of 62 renal transplant recipients at a tertiary care center in El Paso, Texas. In our study population, $43 \%$ of patients had a positive urine culture and were treated for a UTI. $30 \%$ of those UTIs were caused by an ESBLproducing organism. Of note, $43 \%$ of the patients who developed a UTI also had a recurrent episode. Notably, one patient had 25 recurrences of UTIs caused by ESBL producing E. coli. K. pneumoniae and E. coli were the most commonly cultured non-ESBL-producing bacteria (40.7\%). E. coli was the most frequently cultured ESBL-producing bacteria (62.5\%), while K. pneumoniae was the second most frequently cultured ESBL-producing bacteria (37.5\%). There were three transplant rejections; two had ESBL UTIs and one had 11 recurrent UTIs prior to rejection. Diabetes, the length of ureteral stents, and higher troughs of immunosuppressant therapy were observed to be correlated with higher rates of UTIs in our patient population. Our findings are consistent with reports published in current literature regarding rates of UTIs among renal transplant recipients. Due to increasing prevalence of multi-drug resistant organisms and increasing prevalence of carbapenem-resistant Enterobacteriaceae (CRE), it becomes imperative for clinicians to include CRE in the differential diagnosis when evaluating renal transplant recipients with recurrent UTIs. More importantly, CRE must be considered when evaluating renal transplant recipients with recurrent UTIs caused by ESBL producing organisms.
\end{abstract}

Keywords: Clinical Features, Outcomes, ESBL, UTI, Renal Transplant

\section{Background}

Multidrug-resistant organisms have become an increasing challenge in the management of both solid and non-solid organ transplants. This problem is especially true with extended-spectrum beta-lactamases (ESBL) and carbapenem-resistant Enterobacteriaceae (CRE) infections. Bloodstream infections are a significant cause of mortality in solid-organ transplantations as well as hematopoietic stem cell transplantations. Gram-positive infections are the most frequent etiologic agents for all transplantations, except in renal transplants (1). Urinary tract infections (UTI) are the leading infectious complication among renal transplant recipients, affecting $5 \%-36 \%$ of patients (2). Gram-negative infections originating from the urinary tract predominate the renal transplant population; Escherichia coli is the most common organism, followed by Pseudomonas aeruginosa, Enterobacter cloacae, Klebsiella pneumoniae, and Klebsiella oxytoca. Of these five, three have been implicated as the most common ESBL-producing or- ganisms in the United States (US) (3-5). Although renal transplant outcomes have steadily improved with the advent of selective immunosuppressive agents, mortality in renal transplantations from bacterial infection has remained static at $57 \%$ for the last decade $(6,7)$. The increasing prevalence of ESBL-producing organisms complicates treatment options for post-renal transplantation infections. Alevizakos et al. reported that 1 in 10 renal transplant recipients get infected with a UTI from an ESBLproducing Enterobacteriaceae (8). ESBL infections are susceptible to aminoglycosides; however, aminoglycosides are potentially nephrotoxic and increase the risk of graft loss (7). Therefore, carbapenems are the antibiotics of choice in treating UTIs caused by ESBL-producing organisms (9).

\section{Objectives}

This study aims to analyze the outcome of renal transplants at a tertiary care center in El Paso, Texas, and identify

Copyright ( ) 2019, International Journal of Infection. This is an open-access article distributed under the terms of the Creative Commons Attribution-NonCommercial 4.0 International License (http://creativecommons.org/licenses/by-nc/4.0/) which permits copy and redistribute the material just in noncommercial usages, provided the original work is properly cited. 
the risk factors associated with renal transplantation associated UTIs by ESBL-producing organisms. This study also aims to assess the rate of UTI recurrence after renal transplant, and postulate the failure of carbapenems as a treatment for recurrent UTIs.

\section{Methods}

We performed a retrospective study of 62 renal transplant recipients at a 327-bed tertiary care medical center in El Paso, TX, between January 2016 and September 2017. The inclusion criteria for this study were patients who received a renal transplant within the mentioned period. The exclusion criteria for this study included patients who did not undergo transplantation at the study center.

All patients reviewed and included in this study received a renal transplant from either a cadaveric or living donor. Urinalysis and cultures were performed on patients who were both symptomatic and asymptomatic. Urine was collected via clean catch and sampled from indwelling catheters. Cultures were performed using a MicroScan and sensitivities were performed via Kirby-Bauer testing and Epsilometer testing. Various pathogens were isolated via routine urinalysis from renal transplant recipients. The patients with cultures positive for ESBL-producing organisms were included as a subgroup for analysis to determine further risk factors associated with ESBL UTIs posttransplant. All patients are part of a renal transplant registry that stores data that will be used for future analysis to monitor patient outcomes.

\subsection{Data Collection}

The following information was retrieved via retrospective analysis from the medical records of the patient's that received renal transplants: type of donor (living or deceased), immunosuppressive therapy, drug prophylaxis, surgical procedures, number of UTIs, graft rejection, type of dialysis prior to transplant, time on dialysis, presence of nephrolithiasis, and presence of diabetes (Table 1). Urine samples were collected from patients by either clean catch or through their indwelling Foley catheter and then cultured in the facility's microbiology department. Renal transplant recipients routinely get a urinalysis (UA) and urine culture every 3 months after transplantation. Patients were treated for an infection if they had signs and symptoms such as fever, dysuria, urgency, and frequency. If they were asymptomatic, patients were treated if their UA had more than 5-10 white blood cells or if their culture is positive.

\section{Results}

Between January 2016 and September 2017, 62 patients received a renal transplant. $68 \%$ of patients were male (42/62); 32\% of patients were female (20/62). The mean age of the sample population was 56 years old (range: 25 - 73 years old). The majority of donations came from deceased donors (90.3\%); the status of cadaveric donors versus direct organ donation is unknown. Only six patients (9.6\%) received transplants from living donors.

Of the 62 patients who received a renal transplant, $43 \%$ had a positive urine culture and were treated for a UTI (27/62). Eight (30\%) of those patients had UTIs with culturepositive ESBL-producing organisms. As depicted in Figure 1, Klebsiella pneumoniae and Escherichia coli were the most frequently isolated pathogens with each infecting $40.7 \%$ of patients on either initial or recurrent UTI (11/27). Mixed flora were isolated from $18.5 \%$ of patients (5/27), and Enterococcus faecalis was isolated from $14.8 \%$ of patients (4/27). 29.6\% of patients developed UTI's from an ESBLproducing organism infection (8/27), as depicted in Figure 2. Escherichia coli was the most frequently isolated ESBLproducing organism (62.5\%), Klebsiella pneumoniae was second (37.5\%), with Klebsiella planticola (25\%) and Raoutella planticola (12.5\%) being third and fourth, respectively. Of the 27 patients who got a UTI after their renal transplant, 43\% (17/27) developed a recurrent UTI after treatment of the initial infection. ESBL-producing organisms were responsible for 7 of those occurrences. It is important to note that recurrence of UTIs ranged from 2 to 25 episodes and we were not able to accurately quantify UTIs caused by nonESBL-producing organisms.

Patient 1 initially had two separate $P$. aeruginosa infections, followed by four different recurrences of ESBLproducing K. pneumoniae. Patient 2 developed an ESBLproducing E. coli UTI approximately six months after three separate E. coli UTIs. This patient subsequently had three separate occurrences of ESBL-producing E. coli UTI's. Patient 3 had the highest number of recurrences with 23 independent infections with ESBL-producing E. coli despite treatment with meropenem. Patient 4 had three episodes of UTIs with K. pneumoniae. Patient 5 initially had ESBLproducing E. coli, leading to the development of ESBLproducing K. pneumoniae and ESBL-producing K. planticola, respectively. Patient 6 had two separate incidents of ESBLproducing $E$. coli separated by several months in between each infection. Finally, Patient 7 had two UTIs with two different ESBL-producing organisms. Of note, there was only one patient who had a recurrent UTI caused by only an ESBL-producing organism. This patient had two documented UTIs after transplant with ESBL-producing E. coli causing both. 


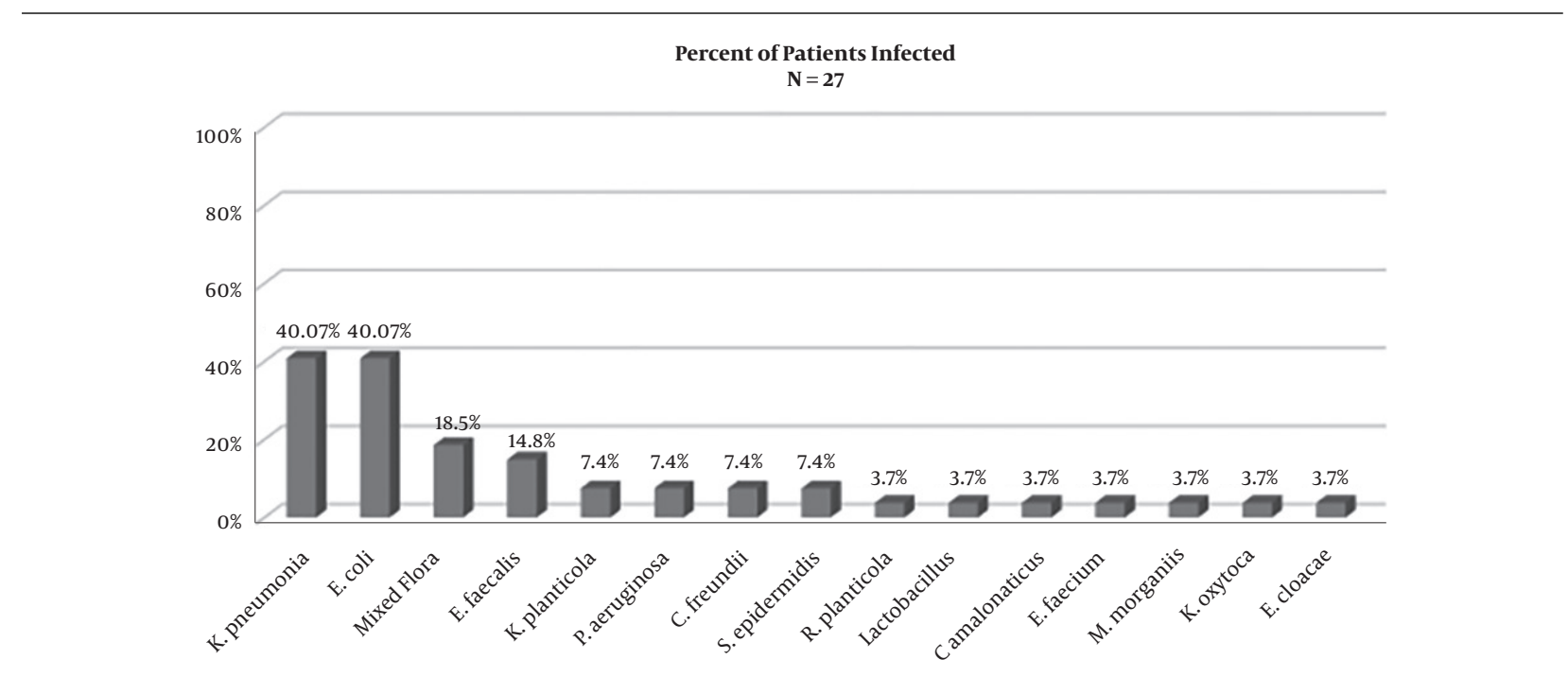

Figure 1. The percent of patients $(n=27)$ infected by each microorganism either as a primary infection or recurrent infection

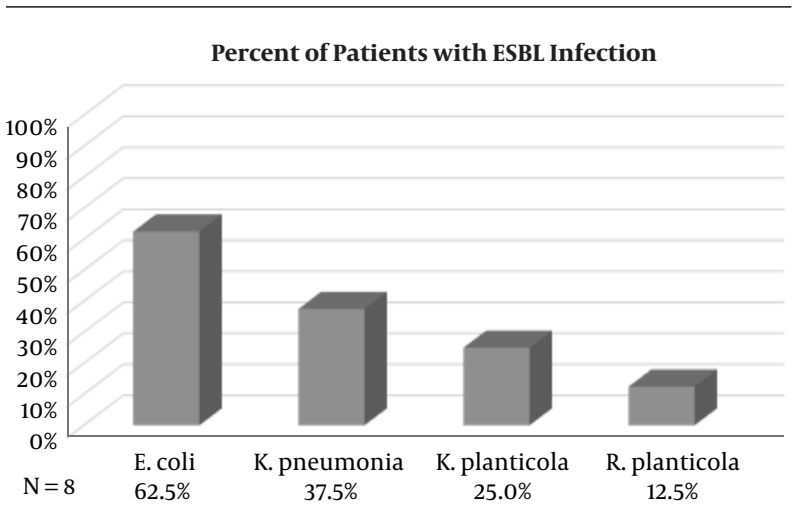

Figure 2. The percent of patients $(\mathrm{n}=8)$ infected by each ESBL-producing organism either as a primary infection or recurrent infection

Notably, there were three transplant rejections out of 62 patients $(4.8 \%)$, all of which were biopsy confirmed cellular rejections. One patient had 11 recurrent episodes of UTIs caused by non-ESBL-producing organisms. This patient also had a history of diabetes and hepatic carcinoma; he subsequently died due to complications secondary to cancer. Two patients had UTIs caused by ESBL-producing organisms. One patient was initially infected with $R$. planticola and then infected with ESBL-producing K. planticola before the rejection. The next patient had a history of diabetes, an open wound with a wound vacuum in place, and multiple UTIs with four episodes of ESBL-producing E. coli before rejection. All 3 of these transplant patients received their transplants from deceased donors. 58\% of transplant recipients had a history of diabetes, and $44 \%$ of those with diabetes had a culture positive UTI after their transplant. $19 \%$ of patients with diabetes had UTIs caused by ESBL-producing organisms, while 25\% had UTIs caused by non-ESBL-producing organisms.

Only 7 patients had a history of nephrolithiasis (11.3\%). $59 \%$ of patients were receiving hemodialysis before transplant. 37\% were receiving peritoneal dialysis. The dialysis status of 1 patient is unknown. All 62 patients received prophylaxis with Valcyte, Fluconazole, and Bactrim and they were on the same immunosuppressive regimen of Tacrolimus, Mycophenolic Acid, and Prednisone. Data on whether immunosuppression regimen was changed before or after the infection is not available. All patients had ureteral stents placed after the transplant that remained in place from 32 to 96 days. All patients received Levofloxacin $500 \mathrm{mg}$ twice a day for 10 days once ureteral stents were removed. In addition to stent removals, 4 patients had renal biopsies, and 1 patient had transplant nephrectomy after stent removal.

\section{Discussion}

Since the first clinical ESBL isolate was identified in Germany in 1983, more than 100 different natural ESBL variants have since been recognized internationally (10). There has been a $14 \%$ increase in ESBL-producing bacteria between 2007 - 2010 (11). ESBLs are described as a group of enzymes with transmissible $\beta$-lactamases encoded by genes that can be exchanged between bacteria via plasmids. These plasmids carry genes that allow the bacteria to break down antibiotics belonging to the cephalosporin and penicillin 
groups, thus rendering the antibiotic ineffective. Currently, the most common genetic variant of ESBL is CTX$\mathrm{M}$, which acquires beta-lactamase genes through a plasmid rather than genetic mutations $(12,13)$. In the United States, the most common ESBL-producing organism is Klebsiella pneumoniae (16\%), followed by Escherichia coli (11.9\%), Klebsiella oxytoca (10\%), and Proteus mirabilis (4.8\%) (14). Previously, ESBL-producing organisms were only identified in nosocomial infections. Reports in the early 2000 s identified actual community-acquired infection or colonization with ESBL-producing E. coli as the etiology for UTI's (15). The lower digestive tract of colonized patients is recognized as the primary source of ESBL-producing organisms. Wilkowski et al. performed a study that showed a robust association between Klebsiella pneumoniae gut colonization and ESBL Klebsiella pneumoniae positive UTI in recipients of renal transplants (16). Factors that facilitate the spread of ESBL include its relative ease of emergence, diversity of parental beta-lactamases, and the mobility of ESBL genes (10). However, the most significant factor driving ESBL evolution has most commonly been attributed to the intense use of third-generation cephalosporins in hospitals $(10,11$, $15,17)$.

There has been a debate about potential risk factors for developing ESBL infections. According to the current data, the risk of developing ESBL-UTI among renal transplant recipients is multifactorial. The most important risk factors include advanced age, length of hospital stay, admission to the intensive care unit, the requirement for ureteral stents or catheters, hemodialysis, and the excessive use of thirdgeneration cephalosporins and aztreonam $(7,10,15,17)$. It is also noted that the total number of antibiotics used, and the longer duration of antibiotic use increased the risk of getting ESBL (17). In a study published by Linares et al., the mean age for ESBL infection post renal transplant was 54 years old and over half the patients were male (55\%) (7). Additionally, 35\% had diabetes, and 57\% had previous use of antibiotics. These reported findings are consistent with our results.

In renal transplant recipients, UTI is the most common infection after transplantation. E. coli is the most common organism isolated, followed by P. aeruginosa, E. cloacae, $K$. pneumoniae, and $K$. oxytoca. Of the five most common organisms, three of them are implicated as the most common ESBL-producing organisms in the US (2-4). Therefore, recipients of renal transplants must be carefully surveilled for infections caused by ESBL-producing bacteria. It is recommended that transplant recipients be screened for UTIs within three months of the operation since untreated UTIs are associated with a higher risk of rejection.

Findings from our study are consistent with the most common bacterial isolates found in renal transplant pa- tients. E. coli and K. pnemoniae were responsible for $40.7 \%$ of UTIs in our transplant recipients. Prior studies demonstrated that with each additional UTI there is an increased risk for recurrence. Anesi et al. reported infections with extended-spectrum cephalosporin-resistant Enterobacteriaceae are associated with developing recurrent UTI within 12 months, compounding the risk of UTI recurrence (18). They also reported that $75 \%$ of ESBL UTIs had a prior history of a UTI with antibiotic treatment. 16 In our study, we found that 7 out of 17 patients had recurrent UTIs with ESBL-producing organisms. Among those patients, three patients had repeated cultures that were consistently positive only for ESBL-producing organisms.

In the studies reviewed, the average rate of ESBL UTI in transplant recipients was $6.9 \%$. There were no reports of graft rejection after UTI. One study provided Bactrim prophylaxis to their patients, similar to our study group (19). These studies demonstrate that the length of stent placement correlates with a higher prevalence of ESBL UTIs and a high rate of UTI recurrence even after stent removal. Immunosuppression with tacrolimus and prednisone were shown to correlate with higher rates of ESBL infections, and higher troughs of tacrolimus and mycophenolate were associated with higher rates of $\operatorname{ESBL} U T I(11,19,20)$. This reported data is consistent with observations from our collected data.

The data collected from our study was similar to the data published by Linares et al. (7). The rate of ESBL infections in our renal transplant patients increased with age, with the average age being 56 years old with a male predominance (69.9\%). Additionally, 58\% of patients from our tertiary care center had diabetes. The presence of diabetes plays an interesting role in the development of UTI in transplant recipients. Diabetes decreases cytokine secretion in the urinary tract, which leads to a reduction in local immunity and serves as a nidus for the growth of multi-drug resistant bacteria, such as ESBL-producing organisms (11). Consistent with the published data, more patients receiving hemodialysis (59\%) had ESBL compared to those receiving peritoneal dialysis. The risk of developing ESBL in kidneys from living donors relative to deceased donors could not be determined due to lack of available data on living donors.

Ureteral stricture is one of the most common complications post-renal transplant. Transplant recipients routinely have percutaneous stents placed to relieve the stricture. The length of stent placement varies among patients. These stents may serves as a focal point for biofilm production. Biofilm provide the perfect environment for bacterial growth and higher survival rates due to poor antibiotic penetration. Additionally, the proximity of various microbial genes allows for the exchange of antibiotic resis- 
tance plasmids. This dynamic environment may explain why ESBL infections increase with an increasing number of days patients are required to have a ureteral stent in place.

Transplant recipients may be more susceptible to infections with multi-drug resistant pathogens because they are more likely to come into contact with these pathogens through their frequent visits to healthcare facilities. The cross-transmission of ESBL-producing organisms between patients within the same hospital may be related to poor hand hygiene among medical personnel or contamination of various diagnostics supplies, such as thermometers or gel used in ultrasonography (10).

Our study demonstrated several patients had recurrent UTIs with ESBL-producing organisms. Of the seven patients noted, each patient had on average more than three recurrent infections, with one patient having 23 recurrent UTIs with ESBL E. coli. This may be attributed to the failure of carbapenems in certain ESBL strains known as Carbapenem-resistant Enterobacteriaceae (CRE). CRE is not detected in routine lab cultures, and the lack of awareness of such pathogens may lead to an increase in infections among individual patients. ESBL-producing organisms can acquire carbapenem resistance by a variety of proposed mechanisms. It can gain resistance to carbapenems by the combination of porin loss and the presence of the plasmid mediated-lactamases, as demonstrated in clinical isolates of Klebsiella pneumoniae exhibiting carbapenem resistance. Additionally, the presence of plasmid-mediated beta-lactamase is capable of hydrolyzing carbapenems. Lastly, these organisms can change in the affinity of penicillin-binding proteins for carbapenems, rendering carbapenems ineffective (15). In a particular case, eight patients with carbapenem resistance mediated by the combination of porin loss and the enzyme deactivation were identified in a single intensive care unit. All eight patients had been treated with imipenem previously. Since no other antibiotic options were available, six of the eight patients died from the infection. In such cases, the use of tigecycline or polymyxins has been proposed for consideration in the treatment of carbapenemresistant Klebsiella (15).

\subsection{Conclusions}

Our findings are consistent with reports published in current literature regarding rates of UTIs among renal transplant recipients. Due to increasing prevalence of multi-drug resistant organisms and increasing prevalence of carbapenem-resistant Enterobacteriaceae (CRE), it becomes imperative for clinicians to include CRE in the differential diagnosis when evaluating renal transplant recipients with recurrent UTIs. More importantly, CRE must be considered when evaluating renal transplant recipients with recurrent UTIs caused by ESBL producing organisms. In the institutional where this study was conducted, CRE was not routinely tested due to lack of resources to conduct genomic analysis for CRE organisms the time of the study. This poses a limitation to study since we do not have the prevalence of CRE available in our study. Additionally, the small study sample size is also a limitation of this study. Additional studies evaluating the prevalence of CRE within our study population is suggested to further characterize the rates of recurrent UTIs caused by multi-drug resistant pathogens among renal transplant recipients.

\section{Acknowledgments}

We would like to thank Bleu Knight for her statistical advisement.

\section{Footnotes}

Authors' Contribution: Study concept and design: Suresh J. Antony; acquisition of data: Judith Pozzerle and Leigh Cooper; analysis and interpretation of data: Leigh Cooper; drafting of the manuscript: Joya Singh and Mikhail de Jesus; critical revision of the manuscript for important intellectual content: Joya Singh, Mikhail de Jesus, and Suresh J. Antony; statistical analysis: Bleu Knight; study supervision: Suresh J. Antony and Joya Singh.

Conflict of Interests: There are no conflicts of interest for the authors of this study.

Ethical Approval: This was a retrospective study that did not require any patient consent or IRB approval. All patient identifiers have been adequately removed.

Financial Disclosure: The authors of this study have no financial interests related to the material in the manuscript. Funding/Support: This study did not require funding.

\section{References}

1. Moreno A, Cervera C, Gavalda J, Rovira M, de la Camara R, Jarque I, et al. Bloodstream infections among transplant recipients: Results of a nationwide surveillance in Spain. Am J Transplant. 2007;7(11):2579-86. doi: 10.1111/j.1600-6143.2007.01964.x. [PubMed: 17868067].

2. Pinheiro HS, Mituiassu AM, Carminatti M, Braga AM, Bastos MG. Urinary tract infection caused by extended-spectrum beta-lactamaseproducing bacteria in kidney transplant patients. Transplant Proc. 2010;42(2):486-7. doi: 10.1016/j.transproceed.2010.02.002. [PubMed: 20304172].

3. Pelle G, Vimont S, Levy PP, Hertig A, Ouali N, Chassin C, et al. Acute pyelonephritis represents a risk factor impairing long-term kidney graft function. Am J Transplant. 2007;7(4):899-907. doi: 10.1111/j.16006143.2006.01700.x. [PubMed: 17286620]. 
4. Lee JR, Bang H, Dadhania D, Hartono C, Aull MJ, Satlin M, et al. Independent risk factors for urinary tract infection and for subsequent bacteremia or acute cellular rejection: A single-center report of 1166 kidney allograft recipients. Transplant. 2013;96(8):732-8. doi: 10.1097/TP.ob013e3182a04997. [PubMed: 23917724]. [PubMed Central: PMC3833249].

5. Ariza-Heredia EJ, Beam EN, Lesnick TG, Cosio FG, Kremers WK, Razonable RR. Impact of urinary tract infection on allograft function after kidney transplantation. Clin Transplant. 2014;28(6):683-90. doi: 10.1111/ctr.12366. [PubMed: 24654771].

6. Woodward RS, Kutinova A, Ricci JF, Brennan DC. The incidence and accumulative costs of sepsis and pneumonia before and after renal transplantation in the United States. Transplant. 2004;78:495-6. doi: 10.1097/00007890-200407271-01336.

7. Linares L, Cervera C, Cofan F, Lizaso D, Marco F, Ricart MJ, et al. Risk factors for infection with extended-spectrum and AmpC beta-lactamaseproducing gram-negative rods in renal transplantation. Am J Transplant. 2008;8(5):1000-5. [PubMed: 18727176].

8. Alevizakos M, Nasioudis D, Mylonakis E. Urinary tract infections caused by ESBL-producing Enterobacteriaceae in renal transplant recipients: A systematic review and meta-analysis. Transpl Infect Dis. 2017;19(6). doi: 10.1111/tid.12759. [PubMed: 28803446].

9. Bodro M, Sanclemente G, Lipperheide I, Allali M, Marco F, Bosch J, et al. Impact of antibiotic resistance on the development of recurrent and relapsing symptomatic urinary tract infection in kidney recipients. Am J Transplant. 2015;15(4):1021-7. doi: 10.1111/ajt.13075. [PubMed: 25676738].

10. Gniadkowski M. Evolution and epidemiology of extendedspectrum beta-lactamases (ESBLs) and ESBL-producing microorganisms. Clin Microbiol Infect. 2001;7(11):597-608. doi: 10.1046/j.1198743x.2001.00330.x. [PubMed: 11737084].

11. Ramadas P, Rajendran P, Krishnan P, Bhaskaran M, Molmenti E. Extended-spectrum-beta-lactamase producing bacteria related urinary tract infection in renal transplant recipients and effect on allograft function. Transplant. 2014;98:475. doi: 10.1097/00007890201407151-01583.

12. Winokur PL, Canton R, Casellas JM, Legakis N. Variations in the prevalence of strains expressing an extended-spectrum beta-lactamase phenotype and characterization of isolates from Europe, the Americas, and the Western Pacific region. Clin Infect Dis. 2001;32 Suppl 2:S94-103. doi: 10.1086/320182. [PubMed: 11320450].

13. Brolund A. Overview of ESBL-producing Enterobacteriaceae from a Nordic perspective. Infect Ecol Epidemiol. 2014;4. doi: 10.3402/iee.v4.24555. [PubMed: 25317262]. [PubMed Central: PMC4185132].

14. Castanheira M, Farrell SE, Krause KM, Jones RN, Sader HS. Contemporary diversity of beta-lactamases among Enterobacteriaceae in the nine U.S. census regions and ceftazidime-avibactam activity tested against isolates producing the most prevalent betalactamase groups. Antimicrob Agents Chemother. 2014;58(2):833-8. doi: 10.1128/AAC.01896-13. [PubMed: 24247134]. [PubMed Central PMC3910895].

15. Paterson DL, Bonomo RA. Extended-spectrum beta-lactamases: A clinical update. Clin Microbiol Rev. 2005;18(4):657-86. doi: 10.1128/CMR.18.4.657-686.2005. [PubMed:16223952]. [PubMed Central: PMC1265908].

16. Wilkowski P, Gajko K, Marczak M, Hryniewiecka E, Wojtowicz M, Dobrzaniecka K, et al. Clinical significance of gastrointestinal carriage of klebsiella Pneumoniae-producing extended-spectrum betalactamases in kidney graft recipients. Transplant Proc. 2018;50(6):18747. doi: 10.1016/j.transproceed.2018.03.114. [PubMed:30056919].

17. Lautenbach E, Patel JB, Bilker WB, Edelstein PH, Fishman NO Extended-spectrum beta-lactamase-producing Escherichia coli and Klebsiella pneumoniae: Risk factors for infection and impact of resistance on outcomes. Clin Infect Dis. 2001;32(8):1162-71. doi: 10.1086/319757. [PubMed: 11283805].

18. Anesi JA, Lautenbach E, Nachamkin I, Garrigan C, Bilker WB Omorogbe J, et al. The role of extended-spectrum cephalosporinresistance in recurrent community-onset Enterobacteriaceae urinary tract infections: A retrospective cohort study. BMC Infect Dis 2019;19(1):163. doi: 10.1186/s12879-019-3804-y. [PubMed: 30764770]. [PubMed Central: PMC6376680]

19. Pilmis B, Scemla A, Join-Lambert O, Mamzer MF, Lortholary O, Leg endre $C$, et al. ESBL-producing Enterobacteriaceae-related urinary tract infections in kidney transplant recipients: Incidence and risk factors for recurrence. Infect Dis (Lond). 2015;47(10):714-8. doi: 10.3109/23744235.2015.1051107. [PubMed: 26024285].

20. Brakemeier S, Taxeidi SI, Zukunft B, Schmidt D, Gaedeke J, Durr M, et al. Extended-spectrum beta-lactamase-producing Enterobacteriaceae-related urinary tract infection in kidney transplant recipients: Risk factors, treatment, and long-term outcome. Transplant Proc. 2017;49(8):1757-65. doi: 10.1016/j.transproceed.2017.06.033. [PubMed: 28923621]. 
Table 1. Summary of Patient Demographics

\begin{tabular}{|c|c|c|c|c|c|c|c|}
\hline $\begin{array}{l}\text { Number of UTIs } \\
2017\end{array}$ & Donor Type & $\begin{array}{l}\text { Surgical } \\
\text { Procedures }\end{array}$ & Graft Rejection & $\begin{array}{l}\text { Type of Prior } \\
\text { Dialysis }\end{array}$ & Time on Dialysis & Kidney Stones & Diabetes \\
\hline 11 & $\mathrm{DD}$ & Stent Removal & No & $\begin{array}{l}\text { Continuous cycling } \\
\text { peritoneal dialysis }\end{array}$ & 2192 days & No & No \\
\hline 8 & DD & $\begin{array}{l}\text { Stent removal, } \\
\text { transplant } \\
\text { nephrectomy } \\
10 / 17 / 2017\end{array}$ & No & Hemodialysis & 1278 days & No & Yes \\
\hline 6 & DD & Stent Removal & No & Hemodialysis & 2462 days & No & No \\
\hline 6 & DD & Stent Removal & No & Hemodialysis & 2196 days & No & Yes \\
\hline 5 & DD & Stent Removal & No & $\begin{array}{l}\text { Continuous cycling } \\
\text { peritoneal dialysis }\end{array}$ & 1433 days & No & No \\
\hline 2 & DD & $\begin{array}{l}\text { Stent removal, } \\
\text { kidney biopsy } 3 \\
\text { months later }\end{array}$ & $\begin{array}{l}\text { Cellular BANFF } \\
\text { Grade I-B; BK }\end{array}$ & Hemodialysis & 2158 days & No & No \\
\hline 2 & DD & Stent Removal & No & Hemodialysis & 2259 days & No & No \\
\hline 2 & DD & Stent Removal & No & Hemodialysis & 1965 days & No & No \\
\hline 2 & DD & Stent Removal & No & Hemodialysis & 1991 days & No & Yes \\
\hline 2 & DD & Stent Removal & No & Hemodialysis & 643 days & No & Yes \\
\hline $\mathbf{1}$ & DD & $\begin{array}{l}\text { Kidney Biopsy, Stent } \\
\text { removal } 1 \text { month } \\
\text { post-biopsy }\end{array}$ & No & Hemodialysis & 1745 days & No & Yes \\
\hline $\mathbf{1}$ & DD & Stent Removal & No & Hemodialysis & 590 days & No & Yes \\
\hline $\mathbf{1}$ & DD & $\begin{array}{l}\text { Pt deceased prior to } \\
\text { any procedures }\end{array}$ & No & Hemodialysis & 2317 days & Yes & Yes \\
\hline $\mathbf{1}$ & DD & Stent Removal & No & Hemodialysis & 1271 days & No & No \\
\hline $\mathbf{1}$ & DD & Stent Removal & No & Peritoneal & 1985 days & No & No \\
\hline $\mathbf{1}$ & LD & Stent Removal & No & Peritoneal & 621 days & No & No \\
\hline $\mathbf{1}$ & DD & Stent Removal & No & Hemodialysis & 2252 days & No & No \\
\hline $\mathbf{0}$ & DD & Stent Removal & No & Hemodialysis & 542 days & No & Yes \\
\hline $\mathbf{0}$ & LD & Stent Removal & No & Hemodialysis & 1550 days & No & Yes \\
\hline $\mathbf{0}$ & DD & Stent Removal & No & Peritoneal & 419 days & No & No \\
\hline $\mathbf{0}$ & LD & Stent Removal & No & Hemodialysis & 265 days & No & No \\
\hline $\mathbf{0}$ & DD & Stent Removal & No & Hemodialysis & 1068 days & No & No \\
\hline $\mathbf{0}$ & DD & Stent Removal & No & Hemodialysis & 1143 days & No & No \\
\hline $\mathbf{0}$ & DD & Stent Removal & No & Peritoneal & 5090 days & No & Yes \\
\hline $\begin{array}{l}\text { Number of UTIs } \\
2016\end{array}$ & Donor Type & $\begin{array}{l}\text { Surgical } \\
\text { Procedures }\end{array}$ & Graft Rejection & $\begin{array}{l}\text { Type of Prior } \\
\text { Dialysis }\end{array}$ & Time on Dialysis & Kidney Stones & Diabetes \\
\hline 25 & DD & Stent Removal & No & Hemodialysis & 1783 days & No & Yes \\
\hline 13 & DD & Stent Removal & No & $\begin{array}{l}\text { Continuous cycling } \\
\text { peritoneal dialysis }\end{array}$ & 2213 days & No & No \\
\hline 11 & DD & $\begin{array}{l}\text { Stent removal, } \\
\text { kidney biopsy } 2 \\
\text { months later }\end{array}$ & $\begin{array}{l}\text { Cellular BANFF } \\
\text { Grade I-A; BK }\end{array}$ & $\begin{array}{l}\text { Continuous cycling } \\
\text { peritoneal dialysis }\end{array}$ & 2197 days & No & Yes \\
\hline 10 & DD & Stent Removal & No & Hemodialysis & 1516 days & No & No \\
\hline 8 & DD & Stent Removal & No & Hemodialysis & 2389 days & No & Yes \\
\hline 7 & DD & Stent Removal & No & Hemodialysis & 970 days & No & No \\
\hline 5 & DD & Stent Removal & No & Hemodialysis & 2703 days & No & No \\
\hline 5 & DD & Stent Removal & No & $\begin{array}{l}\text { Continuous cycling } \\
\text { peritoneal dialysis }\end{array}$ & 1437 days & No & Yes \\
\hline 4 & DD & Stent Removal & No & $\begin{array}{l}\text { Continuous cycling } \\
\text { peritoneal dialysis }\end{array}$ & 2110 days & No & Yes \\
\hline 4 & DD & Stent Removal & No & Hemodialysis & 4451 days & No & Yes \\
\hline 3 & DD & Stent Removal & No & None & $\mathrm{N} / \mathrm{A}$ & No & No \\
\hline 2 & DD & Stent Removal & No & Hemodialysis & 1253 days & No & Yes \\
\hline
\end{tabular}




\begin{tabular}{|c|c|c|c|c|c|c|c|}
\hline 2 & DD & Stent Removal & No & $\begin{array}{l}\text { Continuous cycling } \\
\text { peritoneal dialysis }\end{array}$ & 395 days & No & No \\
\hline 1 & DD & Stent Removal & No & Hemodialysis & 1862 days & No & No \\
\hline 1 & DD & Stent Removal & No & Hemodialysis & 2216 days & No & Yes \\
\hline $\mathbf{0}$ & DD & $\begin{array}{l}\text { Stent removal, } \\
\text { kidney biopsy } 3 \\
\text { months later }\end{array}$ & $\begin{array}{l}\text { Cellular BANFF } \\
\text { Grade I-B; BK }\end{array}$ & Hemodialysis & 2485 days & No & Yes \\
\hline $\mathbf{0}$ & DD & Stent Removal & No & $\begin{array}{l}\text { Continuous cycling } \\
\text { peritoneal dialysis }\end{array}$ & 2322 days & No & Yes \\
\hline $\mathbf{0}$ & DD & Stent Removal & No & $\begin{array}{l}\text { Continuous cycling } \\
\text { peritoneal dialysis }\end{array}$ & 2001 days & No & Yes \\
\hline $\mathbf{0}$ & DD & Stent Removal & No & Hemodialysis & 2931 days & No & Yes \\
\hline $\mathbf{0}$ & $\mathrm{DD}$ & Stent Removal & No & $\begin{array}{l}\text { Continuous cycling } \\
\text { peritoneal dialysis }\end{array}$ & 1983 days & No & Yes \\
\hline $\mathbf{0}$ & DD & Stent Removal & No & $\begin{array}{l}\text { Continuous cycling } \\
\text { peritoneal dialysis }\end{array}$ & 763 days & No & Yes \\
\hline $\mathbf{0}$ & DD & Stent Removal & No & Hemodialysis & 2243 days & No & Yes \\
\hline $\mathbf{0}$ & DD & Stent Removal & No & $\begin{array}{l}\text { Continuous cycling } \\
\text { peritoneal dialysis }\end{array}$ & 1720 days & No & No \\
\hline $\mathbf{0}$ & DD & Stent Removal & No & Hemodialysis & 1736 days & No & Yes \\
\hline $\mathbf{0}$ & DD & Stent Removal & No & Hemodialysis & 2504 days & No & Yes \\
\hline $\mathbf{0}$ & DD & Stent Removal & No & $\begin{array}{l}\text { Continuous cycling } \\
\text { peritoneal dialysis }\end{array}$ & 2238 days & No & Yes \\
\hline $\mathbf{0}$ & DD & Stent Removal & No & $\mathrm{N} / \mathrm{A}$ & 1649 days & No & Yes \\
\hline $\mathbf{0}$ & LD & Stent Removal & No & $\begin{array}{l}\text { Continuous cycling } \\
\text { peritoneal dialysis }\end{array}$ & 280 days & No & Yes \\
\hline $\mathbf{0}$ & LD & Stent Removal & No & Hemodialysis & 687 days & No & No \\
\hline $\mathbf{0}$ & DD & Stent Removal & No & Peritoneal & 1538 days & No & Yes \\
\hline $\mathbf{0}$ & DD & Stent Removal & No & Hemodialysis & 3489 days & No & Yes \\
\hline $\mathbf{0}$ & $\mathrm{DD}$ & Stent Removal & No & Peritoneal & 2362 days & No & No \\
\hline $\mathbf{0}$ & DD & Stent Removal & No & Hemodialysis & 771 days & No & No \\
\hline $\mathbf{0}$ & $\mathrm{DD}$ & Stent Removal & No & Peritoneal & 1338 days & No & Yes \\
\hline $\mathbf{0}$ & LD & Stent Removal & No & Peritoneal & 648 days & No & No \\
\hline $\mathbf{0}$ & DD & Stent Removal & No & Peritoneal & 1456 days & No & Yes \\
\hline $\mathbf{0}$ & DD & Stent Removal & No & Hemodialysis & 3294 days & No & Yes \\
\hline $\mathbf{0}$ & $\mathrm{DD}$ & Stent Removal & No & Hemodialysis & 2637 days & No & Yes \\
\hline
\end{tabular}

